\title{
FIRST TESTS ON NEAR REAL TIME ICE TYPE CLASSIFICATION IN ANTARCTICA
}

\author{
Susanne Lehner ${ }^{1}$, Thomas Krumpen ${ }^{2}$, Anja Frost $^{1}$, Rudolf Ressel ${ }^{1}$, Thomas E. Busche ${ }^{1}$, Egbert Schwarz ${ }^{1}$ \\ ${ }^{1}$ German Aerospace Center (Deutsches Zentrum für Luft- und Raumfahrt; DLR) \\ ${ }^{2}$ Alfred Wegener Institute (AWI)
}

\begin{abstract}
In this paper, we explore the capabilities of an algorithm for ice type classification. Our main motivation and exemplary application was the recent incident of the research vessel Akademik Shokalskiy, which was trapped in pack ice for about two weeks. Strong winds had driven ice floes into a bay, forming an area of pack ice, blocking the ship's advancement. High-resolution satellite images helped to assess the ice conditions at the location. To extract relevant information automatically from the images, we apply an algorithm that is aimed to generate an ice chart, outlining the different ice type zones such as pack ice, fast ice, open water. The algorithm is based on texture analysis. Textures are selected that allow recognition of different structures in ice. Subsequently, a neural network performs the classification. Since results are output in near real time, the algorithm offers new opportunities for ship routing in ice infested areas.
\end{abstract}

Index Terms - GLCM features, neural network, ship navigation, TerraSAR-X, sea ice classification

\section{INTRODUCTION}

On December 25, 2013, 74 scientists and tourists were trapped in the Antarctic on board the Russian research vessel Akademik Shokalskiy. Strong winds had driven ice floes into a bay, forming a vast area of pack ice and blocking the ship's advancement. The Chinese icebreaker Xue Long arrived to assist the Akademik Shokalskiy. But it could only get within sight before it also got stuck in the ice. On January 2, 2014, a helicopter was dispatched from the Xue Long to transport the passengers on board the Akademik Shokalskiy to the Australian icebreaker Aurora Australis, standing by in open waters. High-resolution satellite data helped to assess the ice conditions at the location.

In this paper, we present an algorithm that is aimed to generate an ice chart from images acquired by the German radar satellite TerraSAR-X. The algorithm is split up in two steps: In the first step, texture features are extracted from TerraSAR-X images. The extracted texture features allow a more detailed analysis of different structures in ice. In the second step, the texture features are fed into an artificial neural network, which performs the classification of the ice types i.e. pack ice, fast ice, open water. Pack ice class is subdivided into highly and little deformed pack ice, since these deformations are hard to cross, even for icebreakers. Figure 1 outlines the data flow of our algorithm.

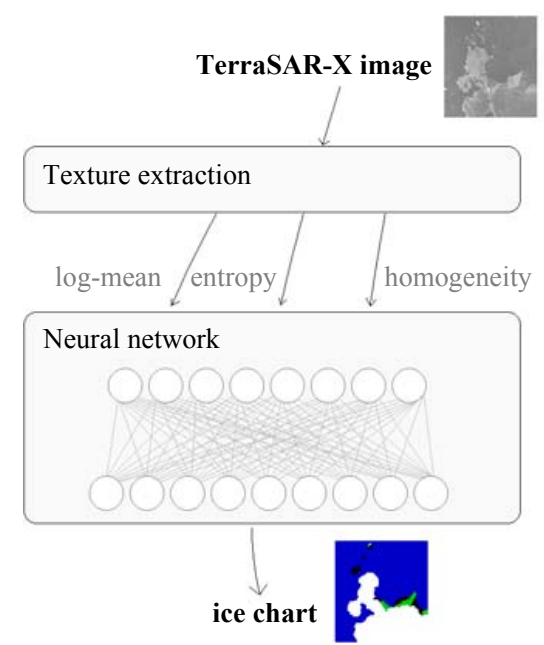

Figure 1: Data flow of the proposed algorithm for near real time ice type classification

Tests on TerraSAR-X images taken over the trapped Akademik Shokalskiy show the selected texture features provide a good basis for distinguishing between ice types. $95.3 \%$ of the illuminated area is classified correctly.

\section{TERRASAR-X TEST SCENE}

Seeing through clouds and darkness, the radar satellite TerraSAR-X is able to observe the ocean and frozen waters from an altitude of approx. 500 kilometers, providing a swathe width of 30 kilometers. To do this, it emits microwaves that are reflected back to the satellite in a way that depends on the characteristics of the reflecting surface. The technology provides an extremely high resolution of down to three meters. This is crucial, as the ice structure may change greatly over just a few hundred meters. The satellite data provides scientists with a high-resolution 
image of the surface of the ice.

Faced with the situation of the research vessel Akademik Shokalskiy, scientists from the DLR Microwaves and Radar Institute used TerraSAR-X to acquire images of the trapped research vessel. Figure 2 shows exemplarily images taken on December 30, 2013. Software at the DLR Research Center for Maritime Safety in Bremen tracked the ship (marked by the squares in Figure 2). Maritime ice researchers from the Alfred Wegener Institute and the EOC analyzed the images and passed on their findings to the rescue center in Australia, the Emergency Response Division at the Australian Maritime Safety Authority.

\section{ICE TYPE CLASSIFICATION}

\subsection{Approach and design concepts}

Several approaches have been explored in the scientific community in order to classify ice types automatically or semi-automatically from SAR images, listed very comprehensively in [1]. Most are based on the extraction of texture features, particularly gray-level co-occurrence matrices (GLCM features) [1][2][3], wavelet based features [4] and Markov random fields [2][5]. Because of the reported suitability [2][3], we use GLCM features in our algorithm.

After texture extraction, one than has to choose whether to use supervised or unsupervised classification. In order to incorporate expert knowledge of the past, we chose super- vised classification. For the classification step, we make use of an artificial neural network [6].

\subsection{Texture extraction}

In our study, we tested a number of moment based and GLCM features: average mean, variance, third moment, fourth moment, log-mean, entropy, homogeneity, contrast, uniformity, and correlation (see formulae in [2] and [7]). The sliding window is $11 \times 11$ pixels with a GLCM displacement vector length of 1 pixel. All four commonly used directions of the displacement vector are used in computations and the respective matrices are combined into one GLCM. The original bit depth of 8 bit was downscaled to a 6 bit gray level image. Since the features which distinguish the ice types are rather coarse, we reduced the resolution of the image by the factor of 6 . Another reason for working on lower resolution is computation speed which is pivotal for a near real time service.

By visual inspection of the different feature images generated from TerraSAR-X scenes, we observed a number of similarities (homogeneity and uniformity, entropy and contrast). The most insightful features prove to be logmean, entropy, and homogeneity. In the following, we focus on these three features.

The Figures 3-5 show the GLCM features log-mean, entropy and homogeneity extracted from the TerraSAR-X images in Figure 2. One can clearly see that the features differ in each ice type zone: In pack ice, high entropy values



Figure 2: TerraSAR-X images taken on December 30, 2013. The images altogether cover $100 \mathrm{x} 50 \mathrm{~km}$. All around the ships Akademik Shokalskiy and Xue Long, there is pack ice, which appears bright due to its rough surface. On the left of the pack ice, there is smooth fast ice. Open water is located on the right. 


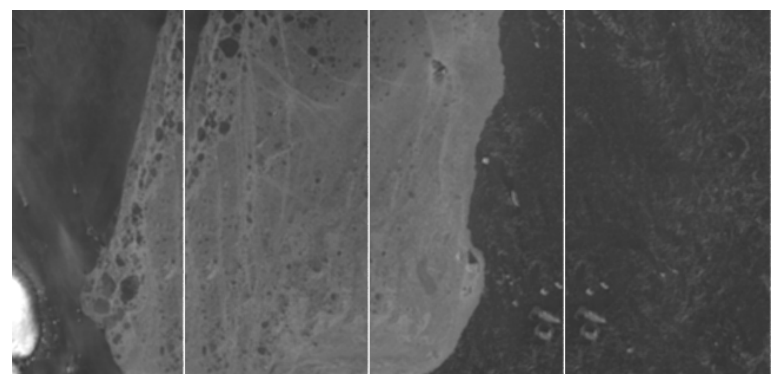

Figure 3: Log-mean extracted from Figure 2. A higher log-mean value is displayed by a lighter gray.

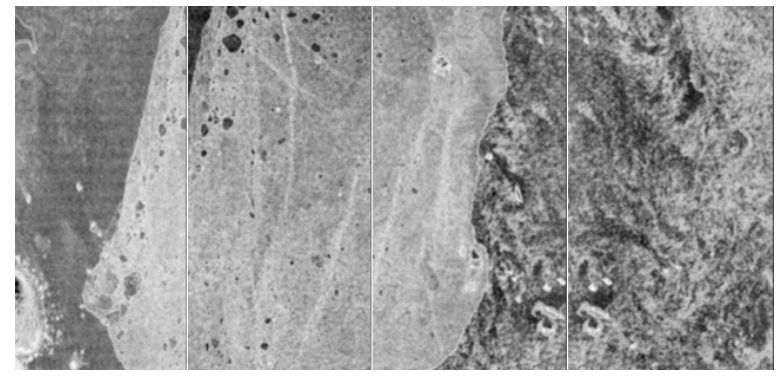

Figure 4: GLCM entropy extracted from Figure 2. A higher entropy value is displayed by a lighter gray.

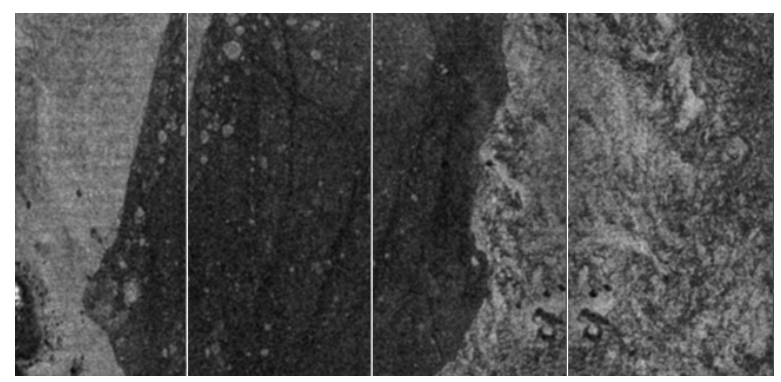

Figure 5: GLCM homogeneity extracted from Figure 2. A higher homogeneity value is displayed by a lighter gray.

predominate, while the homogeneity is low. In fast ice, both the entropy and the homogeneity are neither high nor low, but uniform. By contrast with fast ice, the open water zone shows more variations in entropy and homogeneity values. In addition, the log-mean is slightly lower than in fast ice. Pack ice indicates the highest log-mean.

\subsection{Neural network}

For the artificial neural network, we rely on the popular and well-tested FANN library [6]. We use 10 input neurons, two hidden layers with 8 and 9 neurons, respectively. Training procedure is RPROP. The number of output neurons depends on the number of classes. For the scene presented in this paper, we use neurons for each of the following ice types: fast ice, highly deformed pack ice, little deformed pack ice, and open water with high as well as low ice concentration. Land ice was visible in the scene yet was not used in the classification, since it is irrelevant once land masking is applied. FANN output is shown in Figure 6. As can be seen, it coincides well with the real ice conditions noted in Figure 2. Only small parts of the images are misclassified: The left margin of the open water zone is classified as fast ice. The fast ice zone is speckled with "ponds" of open water and pack ice in FANN output.

\subsection{Quantitative evaluation}

In order to express the accuracy of the generated ice chart quantitatively, we recorded the number of misclassified pixels and the number of correctly classified pixels (separately for each of the three ice type zone noted in Figure 2). Table 1 lists the results. As can be seen, pack ice is recognized most accurately. Here, $97.7 \%$ of the pixels are classified correctly. The whole test scene coincides with the real ice situation for $95.3 \%$ of the illuminated area.

It should be pointed out that the amount of correctly classified pixels is high, as the data used for training the neural network derive from the same image - though only a small part of it $(2.7 \%)$ has been selected for training. By generating ice charts from other images without adding new training data, an increase of the misclassified area might be expected.

Table 1: Quantitative analysis of the resulting ice chart shown in Figure 6

\begin{tabular}{|l||c|}
\hline ice type zone & correctly classified area \\
\hline \hline fast ice & $90.1 \%$ \\
\hline $\begin{array}{l}\text { pack ice (highly and little } \\
\text { deformed) }\end{array}$ & $97.7 \%$ \\
\hline $\begin{array}{l}\text { open water (with high and } \\
\text { low ice concentration) }\end{array}$ & $94,9 \%$ \\
\hline \hline whole test scene & $95.3 \%$ \\
\hline
\end{tabular}

\subsection{Computing time}

All data were processed on a $2.7 \mathrm{GHz}$ single core CPU. The computing time for generating the ice chart from one image amounts to approximately 20 minutes, i.e. processing the whole scene depicted in Figure 2 requires 80 minutes in sequencial processing. In future works, we want to carry out parallel processing.

\section{CONCLUSION}

In this paper, we have shown first test results of an algorithm for ice type classification using TerraSAR-X images. By the selection of significant texture features, distinct structures in different ice type zones are revealed. An artificial neural network performs the classification and outputs an ice chart, in which different ice type zones are 


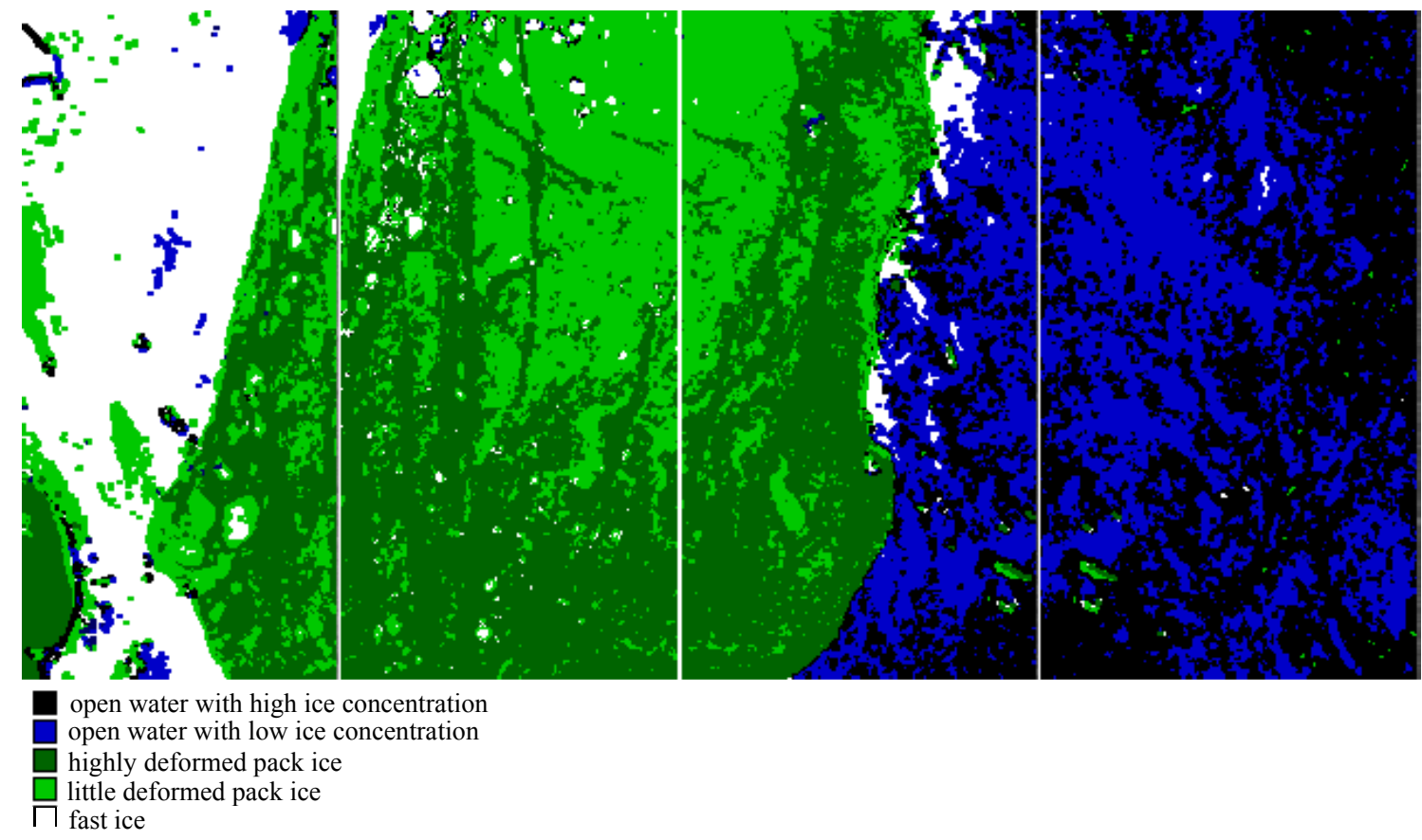

Figure 6: Resulting ice chart generated from the images shown in Figure 3, 4 and 5 using the FANN library. Obviously, the chart is close to the real ice situation noted in Figure 2.

outlined. In our tests, less than five per cent of the illuminated area is misclassified. Therefore, the proposed TerraSAR-X based classification promises powerful assistance for ship routing in Antarctic waters.

In future works, we plan to additionally flesh out some unsupervised classification based on the GLCM features to systematize such hands-on visual inspection.

\section{REFERENCES}

[1] N. Zakhvatkina, V. Alexandrov, O. Johannessen, S. Sandven, and I. Frolov, "Classification of Sea Ice Types in ENVISAT Synthetic Aperture Radar Images," Geoscience and Remote Sensing, IEEE Transactions on, 51(5), 1648-1664, 2013.

[2] D. Clausi, "Comparison and fusion of co-occurrence, Gabor and MRF texture features for classification of SAR sea-ice imagery," Atmosphere-Ocean, 39(3), pp. 183-194, 2001.

[3] A. Bogdanov, S. Sandven, O. Johannessen, V. Alexandrov, , and L. Bobylev, "Multisensor Approach to Automated Classification of Sea Ice Image Data," Geoscience and Remote Sensing, IEEE Transactions on, 43(7), pp. 1648-1664, 2005.

[4] Q. Yu, C. Moloney, and F. M. Williams. "SAR sea-ice texture classification using discrete wavelet transform based methods," Geoscience and Remote Sensing Symposium, IGARSS'02, IEEE International, 2002.

[5] T. Wang, X. Yang, Y. Wang, J. Fang, and L. Jia, "A multilevel SAR sea ice image classification method by incorporating egg-code-based expert knowledge." Image and Signal Processing (CISP), 5th International Congress on. IEEE, 2012.

[6] S. Nissen, Implementation of a fast artificial neural network library (FANN), Report, Department of Computer Science University of Copenhagen (DIKU) 31, 2003.

[7] R. M. Haralick, K. Shanmugam, and I. H. Dinstein. "Textural features for image classification," Systems, Man and Cybernetics, IEEE Transactions on, (6), pp. 610-621, 1973. 